Revista Calidad en la Educación Superior

Programa de Autoevaluación Académica

Universidad Estatal a Distancia

ISSN 1659-4703

Costa Rica

revistacalidad@uned.ac.cr

\title{
OLIMPIADAS COSTARRICENSES DE CIENCIAS BIOLÓGICAS COMO PROCESO FUNDAMENTAL EN EL MEJORAMIENTO DE LA CALIDAD EDUCATIVA SECUNDARIA DE COSTA RICA EN EL ÁREA DE LA BIOLOGÍA
}

\section{COSTA RICAN BIOLOGICAL SCIENCES OLYMPICS AS A FUNDAMENTAL PROCESS IN IMPROVING SECONDARY EDUCATION QUALITY IN COSTA RICA IN THE AREA OF BIOLOGY}

\author{
Magaly Rodríguez Calvo ${ }^{1}$ \\ marodriguez@uned.ac.cr \\ Universidad Estatal a Distancia, Costa Rica \\ VI Edición \\ Volumen III, Número 2 \\ Noviembre 2012 \\ pp. $122-140$
}

Recibido: febrero, 2012

Aprobado: Junio, 2012

1'Licda. en Biología Tropical, M.S.c. en Administración Educativa de la Escuela de Ciencias Exactas y Naturales, Universidad Estatal a Distancia (UNED). 


\section{Resumen}

Las Olimpiadas Costarricenses de Ciencias Biológicas (OLICOCIBI) potencian el nivel cognitivo mediante competencias académicas dirigidas a estudiantes y docentes de secundaria, donde se optimiza el proceso de enseñanza aprendizaje mediante la aplicación biotecnológica de la biología. Parte de los objetivos es que la población académica segundaria, mediante este tipo de actividades, pueda fortalecer y mejorar la educación científica en Costa Rica.

Estas olimpiadas promueven el estudio activo, participativo y significativo de las ciencias biológicas a nivel de la enseñanza media y preuniversitaria. Además, es una actividad que abre espacios de participación a todas los centros educativos, tanto públicos, como privados, y abarca todas las sedes regionales del país. Estas justas se dividen en dos categorías; la A para estudiantes de X, XI y XII nivel y la B para estudiantes de VIII, IX y X nivel.

La organización de este evento requiere de la estructuración y coordinación de un trabajo integral y en equipo de varias instancias del gobierno como lo son: Universidad Nacional (UNA), Universidad Estatal a Distancia (UNED), Universidad de Costa Rica (UCR), Ministerio de Ciencia y Tecnología (MICIT), Ministerio de Educación Pública (MEP) y otras instituciones emblemáticas en el campo social, científico y biológico del país.

Los antecedentes de OLICOCIBI se iniciaron en el 2007 y la recalcada labor que se ha realizado año tras año, ha impulsado la participación de Costa Rica en la Olimpiada Iberoamericana de Biología (OIAB), obteniendo desde el año 2008 destacados resultados como medallas de bronce, plata y oro, logrando posicionar a Costa Rica en los más altos niveles cognitivos. Es por ello que esta actividad fue declarada de interés institucional por las Universidades Públicas, y de interés educativo por el Ministerio de Educación.

Palabras claves: Olimpiadas de Biología, educación secundaria, calidad educativa, estrategias metodológicas, procesos de enseñanza y aprendizaje, docentes y estudiantes.

\section{Abstract}

The Costa Rican Biological Sciences Olympics (OLICOCIBI), enhance the cognition level through academic competitions directed by students and high school teachers, where the teaching learning process is optimized through the biotechnology application of biology. Part of the goal is that the academic high school population can increase and develop a better scientific education in Costa Rica with those kinds of activities. 
The Olympics promote an active, participatory and significant study method of biological sciences when it comes to middle or secondary and pre-university education. Also, is an activity that opens up alcoves of participation to every single educational school both public and private ones, as well as regional headquarters of the country. The same are divided in two categories; A for students of X, XI, XII level and B for students of VII, IX and X level.

The organization of this event, requires the structuring and coordination of an integral unity and a team of several government agencies such as: Universidad Nacional (UNA), Universidad Estatal a Distancia (UNED), Universidad de Costa Rica (UCR), Ministerio de Ciencia y Tecnología (MICIT) Ministeria de Educación Pública (MEP) and other emblematic institutions from the social, scientific and biological field.

The background of OLICOCIBI stared in 2007 and the crimped work that has been done year after year, has increase the partaking of Costa Rica in the Iberoamerican Biology Olympics (OIAB); acquiring since the year of 2008, distinguish results like bronze, silver and gold medals, achieving to put Costa Rica as one of the highest cognitive levels; therefore, this type of activity was declared as an institutional interest by Public Universities and also as an education interest by the Ministry of Education (MEP).

Keywords: Biology olympics, high school education, education quality, methodological strategies, teaching and learning process, teachers and students.

\section{Introducción}

Las Olimpiadas Costarricenses de Ciencias Biológicas (OLICOCIBI) se realizaron por primera vez en el año 2007, bajo el nombre de I Olimpiada Costarricense de Biología, para ese año la Ministra de Ciencia y Tecnología, Dra. Eugenia Flores Vindas, mostró interés en que se organizaran en Costa Rica las Olimpiadas de Biología. Donde la Universidad Nacional de Costa Rica (UNA) tomó el compromiso de organizar dicho evento, coordinado por la Facultad de Ciencias Exactas y Naturales y la Escuela de Ciencias Biológicas, y fue así como inició este deseo, siendo parte de un proyecto de graduación de dos estudiantes de la Universidad Nacional, quienes fueron apoyados técnica y científicamente por académicos de la Escuela de Ciencias Biológicas, y con patrocinio del Ministerio de Ciencia y Tecnología (Escuela de Ciencias Biológicas, 2007). 
Para el año 2008, la II OLICOCIBI es organizada y ejecutada por la Escuela de Ciencias Biológicas de la UNA, logrando convertirse en una actividad bastante aceptada por los participantes, representando una opción relevante en el intercambio de experiencias y conocimientos del quehacer humano y científico en la educación secundaria de Costa Rica, así como su ejecución a nivel nacional y su afianzamiento como un espacio de amplio interés y proyección para la sociedad costarricense.

Es a partir del año 2008 donde Costa Rica decide participar y competir a nivel internacional en las Olimpiadas Iberoamericanas de Biología (OIAB), siendo un logro importante para el país, ya que obtuvo una medalla de bronce. Esto hace establecer mejores puentes de comunicación entre el sistema de educación secundario y el sistema de estudios superiores, promoviendo la participación costarricense en el ámbito internacional, para lograr aumentar el estímulo de los participantes en procesos cognitivos de la ciencias biológicas, y de esta manera, OLICOCIBI, desde sus inicios y hasta la actualidad, llega a formar parte de una estrategia metodológica que se aplica en la educación secundaria costarricense para fortalecer nuestro sistema educativo en el área científico-tecnológico.

Las Olimpiadas de Biología motivan al estudio activo, participativo y significativo de las ciencias biológicas a nivel de enseñanza media y preuniversitaria, con el fin de contribuir con el desarrollo científico y tecnológico de Costa Rica. Además, abre espacios de participación a todos los centros educativos, tanto públicos, como privados de cualquier parte del país. Por lo tanto, estas olimpiadas concentran diversos grupos de estudiantes que compartirán de manera cognitiva para poner en práctica los conocimientos adquiridos. 
Este tipo de competencias, como las Olimpiadas de Biología, son de gran importancia para la educación secundaria, ya que enriquecen el área de las ciencias, consiguiendo que tanto los estudiantes como los docentes participen, interactúen, intercambien ideas, complementen los conocimientos teóricos con actividades prácticas, para lograr fortalecer la calidad educativa en el sistema secundario costarricense.

El artículo está dirigido a todas las instituciones educativas secundarias, especialmente a los docentes del área de las ciencias biológicas, asimismo, a los estudiantes que tienen mayor interés en esta asignatura, para que logren aumentar su motivación en estas competencias tanto nacionales como internacionales, ya que la participación en estas justas es un valor agregado para adquirir mayor conocimientos biológicos, vinculando tanto la ciencia como la tecnología.

\section{Antecedentes}

Parte de la propuesta de iniciar con este proyecto de extensión, denominado Olimpiadas Costarricenses de Ciencias Biológicas en Costa Rica, se da por el hecho de ser un país que promueve, fortalece y desarrolla una actitud activa y responsable en estudiantes que se enfoquen hacia los avances científicos y tecnológicos en el campo de la biología.

A nivel internacional la Olimpiada Internacional de Biología (IBO) realiza una competencia anual para estudiantes de secundaria, y las primeras se realizaron en los años 60, y actualmente participan más de 70 países de los cinco continentes. La sede central de estas justas internacionales se localiza en República Checa, y cada año se celebra en uno de los países asociados. Cabe mencionar que la primera competencia internacional de Biología se dio entre Checoslovaquia y Polonia (Escuela de Ciencias Biológicas, 2007). 
Se puede decir que las competencias a nivel de Olimpiadas Biológicas en diversos países del mundo han sido de gran tradición y relevancia, incluso países de Latinoamérica como Argentina y México compiten con excelentes niveles de organización, no solo a nivel nacional, sino internacional.

Una vez que Costa Rica inicia este tipo de competencias a nivel nacional en el 2007, decide un año después en el 2008 incorporarse a las competencias de Olimpiadas Iberoamericanas de Biología (OIAB), las cuales se llevaron a cabo la primera vez en el año 2007 en la Ciudad de México (Escuela de Ciencias Biológicas, 2008).

Para el año 2008, se compite por primera vez a nivel lberoamericano, llevándose a cabo estas disputas en el país de Brasil, se contó con una delegación costarricense conformada por académicos del comité organizador del Proyecto de OLICOCIBI y dos estudiantes ganadores de la I Olimpiada Nacional Costarricense de Biología, para esta primera participación se obtuvo una medalla de bronce. Sin duda, esta fue una experiencia constructiva y enriquecedora en el área de la Biología para Costa Rica, donde se compartió cultural, social, educativa y científicamente con delegaciones de países hermanos (Olimpiadas Costarricenses de Ciencias Biológicas (Sin fecha).

\section{Olimpiadas de Ciencias Biológicas versus calidad educativa secundaria}

Este proyecto de extensión tiene entre sus objetivos que tanto los estudiantes como los profesores de la educación secundaria de todo el país puedan potenciar el área biológica como parte de la formación de enseñanza y aprendizaje, mediante el desarrollo de habilidades cognitivas y actitudinales, así como crear espacios de capacitación y actualización en los docentes.

Durante el 2008, la consolidación de la I Olimpiada Costarricense de Biología, a través de la II Olimpiada Costarricense de Ciencias Biológicas a nivel nacional, dio 
la apertura del Programa de Olimpiadas Costarricenses de Ciencias Biológicas a los 21 circuitos regionales educativos del Ministerio de Educación Pública (MEP), proponiéndose dos categorías de nivel para los estudiantes, la categoría $A$ para estudiantes de $\mathrm{X}, \mathrm{XI}$ y XII nivel y el establecimiento de la categoría B para estudiantes de VIII, IX y X nivel (Escuela de Ciencias Biológicas, 2009).

El incluir todos los niveles de secundaria brinda la oportunidad a la mayoría de estudiantes de ser parte de una comunidad estudiantil preparada e identificada con potenciales hacia el área biológica, así como incentivarlos al estudio de la misma. Este proyecto motiva a organizar una actividad de gran relevancia institucional, regional y nacional, la cual inicio en la Universidad Nacional, como una actividad de integración entre la docencia, investigación y extensión, la cual cuenta actualmente con el apoyo de otros entes gubernamentales como MICIT, MEP y de algunas empresas privadas y, desde el 2010, se incorporan al equipo de trabajo la Universidad Estatal a Distancia y la Universidad de Costa Rica, para consolidarse y generar estrategias futuras de participación nacional e internacional.

Debido al impacto positivo que ha generado la OLICICIBI en la sociedad costarricense y en nuestro sistema educativo secundario, donde se le brinda un espacio competitivo a los estudiantes talentosos en el área de las ciencias biológicas, se impulsan las vocaciones científicas y se enriquece la labor del docente, se logró también que las Olimpiadas de Biología se declararan de Interés Institucional en cada universidad participante y de Interés Educativo para el MEP.

Según Pereira (2011), desde el año 2007, cuando inicio este proyecto de extensión hasta el año 2011, se registran un gran aumento de participación tanto de instituciones educativas como de estudiantes, lo cual se evidencia en la siguiente figura. (Figura 1). 
Figura 1. Cantidad de centros educativos y estudiantes participantes en las ediciones 2017 a 2011 de la OLICOCIBI

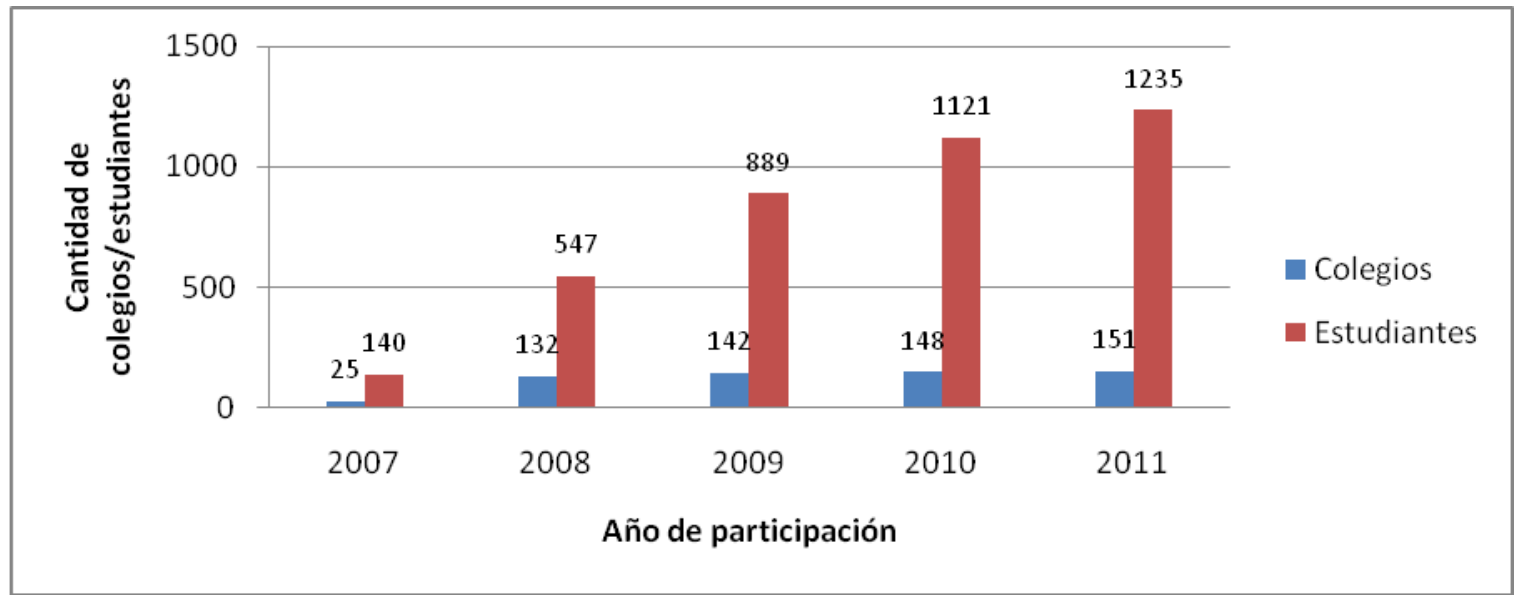

Fuente: Pereira 2011.

OLICOCIBI logra el fortalecimiento y actualización de conocimientos biológicos a docentes y estudiantes involucrados en la enseñanza de la biología en la educación secundaria mediante la realización de capacitaciones o talleres, y por tanto, favorece el aprendizaje significativo para alcanzar una mejor calidad educativa. El desarrollo de estas actividades a docentes de zonas urbanas como de rurales promueve la creatividad en los profesores a la hora de aplicar la mediación pedagógica en sus estudiantes, favoreciendo cada vez más una buena calidad en el proceso educativo.

Este proyecto de extensión denominado Olimpiadas de Biología tiene un gran vínculo con el MEP, porque los contenidos que se desarrollan en las pruebas que se aplican a los estudiantes participantes, son casi los mismos contenidos que tiene el MEP propuestos para cada año o nivel educativo secundario. Lo expuesto anteriormente se confirma con lo indicado por Camacho (2011), la OLICICIBI extiende la valoración cognitiva más allá de los contenidos curriculares establecidos por el MEP, incluyendo en sus evaluaciones aproximadamente un $20 \%$ de contenidos no abarcados en los temarios oficiales del MEP, ya que se incorporan prácticas en el campo, exposiciones y elaboraciones de artículos 
científicos y, además, brinda espacios de capacitación docente, de modo que mejora y diversifica la calidad de la educación biológica.

Por lo tanto, estas competencias logran profundizar y aumentar los conocimientos de los participantes en el área de las ciencias biológicas, de esta manera, los estudiantes se pueden ver beneficiados cuando aplican las pruebas finales de biología en el colegio y, especialmente cuando realizan los exámenes de bachillerato para optar por su grado de educación media. Como consecuencia, dicho proyecto promueve a mejorar promedios en las ramas de la biología cuando ingresen a la Universidad.

Para Camacho (2011), las Olimpiadas de Biología tienen un valor agregado en la educación secundaria costarricense como actividad alfabetizadora en las ciencias biológicas. Permite identificar estudiantes con potencial y facultades meritorias, tanto en el estudio de las ciencias biológicas y sus múltiples ramas, como en la creatividad, perseverancia y capacidad analítica. Asimismo, brinda al profesor la oportunidad de socializar, compartir, discutir e incorporar nuevas estrategias metodológicas en la enseñanza de esta disciplina, fomentando su progreso en los centros educativos.

Las Olimpiadas de Biología, además de lograr que los estudiantes tengan interés por las ciencias biológicas, le proporcionan estrategias de aprendizaje que los lleven a ser reflexivos, críticos, analíticos, creativos, tengan capacidad de observación y puedan interpretar la información.

De acuerdo a lo comentado anteriormente los autores Soler, L; Fleota. A; Carenton, J; Cuello, P; Pérez, P y Palacios, M. (2006), señalan que:

El papel fundamental del docente es gestionar, guiar y enseñar contenidos $y$ procedimientos en el aula, porque es quien más cerca se encuentra de la formación de los estudiantes. Por lo tanto, es responsable de desarrollar diariamente estrategias pedagógicas que les permitan enfrentar la diversidad de 
alumnos (as) y entre ellos, diferenciar aquéllos que tienen afinidad y están motivados en aprender más sobre un contenido específico.

Es importante resaltar que la participación de la comunidad educativa en estas competencias académicas permite concentrar a diversos grupos de estudiantes y docentes que comparten experiencias significativas y cognitivas de aprendizaje en la biología. También estrechan lazos de amistad, responsabilidad, unión de grupo, solidaridad y respeto, así como otros valores, creencias y normas indispensables para el pensamiento crítico científico. Esto provoca en los estudiantes y docentes, el fortalecimiento y desarrollo de una actitud responsable hacia los avances científicos y tecnológicos en el campo de la ciencia.

Para la autora Camacho (2011), las Olimpiadas de Biología consiguen que los estudiantes desarrollen aspectos conceptuales, procedimentales y actitudinales, los cuales contribuyen al mejoramiento de la comunidad científica y tecnológica en el campo de las ciencias biológicas del país. Con respecto a la dimensión conceptual, se determina un refuerzo y aumento de conocimientos biológicos, mientras que para la dimensión procedimental, se visualizó en los contenidos prácticos o de campo, donde los estudiantes obtienen buenos niveles de interpretación de los instrumentos metodológicos y por ende, asimilan y ejecutan los procedimientos biológicos. En el aspecto actitudinal, se determinó que los estudiantes tienen gran interés a las clases de biología, y para ello influye la motivación académica tanto propia como de la institución.

El que los estudiantes logren ampliar sus conocimientos en el área de la biología conlleva a la idea de que están adquiriendo, por parte de los docentes, adecuadas estrategias metodológicas, que les permite asimilar el aprendizaje apropiadamente y mejorar la calidad educativa, es por ello que para las Olimpiadas Costarricenses de Ciencias Biológicas (Sin fecha), sus propósitos son los siguientes: 
a) Establecer puentes de comunicación entre el sistema de educación secundaria con el sistema de estudios superiores.

b) Estimular en los participantes los procesos cognoscitivos de las ciencias biológicas, según la integralidad de los ejes transversales, durante la fase experimental.

c) Formular un programa de capacitación para los estudiantes que representarían a Costa Rica en las Olimpiadas Iberoamericanas de Biología, en coordinación con distintos académicos de las universidades participantes.

d) Promover la participación y el excelente desempeño de Costa Rica en eventos Olímpicos de Biología a nivel internacional.

e) Fomentar el intercambio de experiencias de profesores de biología como una práctica de retroalimentar sus técnicas metodológicas de la enseñanza y aprendizaje de esta disciplina, por medio de los encuentros académicos, colaborando al mejoramiento de los procesos educativos relacionados con las ciencias biológicas en secundaria.

f) Promover e incentivar en los participantes una actitud crítica y responsable hacia los avances científicos y tecnológicos en el campo de las ciencias biológicas, así como la importancia del uso racional de los recursos naturales y de su preservación.

g) Propiciar el intercambio cultural, social y cognitivo, entre estudiantes de diversos centros educativos de todo el país durante el proceso de ejecución de la prueba teórica y práctica final y la clausura nacional de las Olimpiadas Costarricenses de Ciencias Biológicas.

\section{Estrategias metodológicas que se pueden aplicar en las OLICOCIBI}

El proyecto de Olimpiadas de Biología propone utilizar diferentes estrategias metodológicas que logren una adecuada mediación pedagógica en los centros educativos de secundaria de Costa Rica. Para ello, se requiere una constante Revista CAES Vol. III, No.2, Año 2012 ISSN-1659-4703 
innovación en el proceso de enseñanza- aprendizaje, donde tanto los docentes como estudiantes que participan en este evento construyan y adquieran conocimientos conjuntamente y así obtengan experiencias, actitudes y conductas que los lleven a favorecer su proceso de formación. De esta manera, se establece un plano de instrucción a los estudiantes tanto a nivel personal, social y, en un futuro próximo, profesional en el contexto que se deseen especializar.

Actualmente, vivimos en un mundo muy cambiante en el área de la ciencia y tecnología, es por ello que las Olimpiadas de Biología no puede dejar de lado las tecnologías como parte a utilizar en su modelo de enseñanza y aprendizaje hacia los estudiantes y docentes que participan en este proyecto. Al ser la biología una ciencia tan dinámica, se pueden realizar una serie de aspectos como lo son: estudios de caso, análisis de información, problemas a desarrollar, prácticas a ejecutar, interpretar información, trabajar en grupo, entre otras.

Para los autores (Perdomo, 2008; Colegio Hebreo Dr. Jaim Weitzman, sin fecha y Digión; Sosa y Velásquez, 2006), indican que, dependiendo del comportamiento de cada estudiante, se puede visualizar diferentes modelos de aprendizaje que se apoyan en la tecnología para ser utilizados de distinta forma, como lo son: resolver problemas, trabajar independientemente, trabajar en grupo, revisión de información, interpretar datos, confrontar varias informaciones, relacionar otros conocimientos, aplicar conocimientos adquiridos previamente a nuevas situaciones, razonar, aprender, analizar, criticar, desarrollar creatividad constante, observar, comparar, encontrar similitudes y diferencias, realizar hipótesis, deducir, inferir, entre otros. Por lo tanto, para el proyecto de Olimpiadas de Biología el utilizar herramientas tecnológicas tales como computadoras portátiles, microscopios, estereoscopios, otros equipos de laboratorio, videos, proyecciones, entre otros, son estrategias metodológicas que se emplean en las capacitaciones que se le brindan tanto a los estudiantes como docentes para lograr obtener un mayor dinamismo en los procesos de aprendizaje. 
además, de esta manera los docentes consiguen mejorar las competencias pedagógicas. Así, obtendrán éxito y calidad en el proceso académico de la secundaria, sin dejar de lado la integración entre estudiante - estudiante y estudiante - docente para que conjuntamente construyan su aprendizaje por medio de habilidades y estrategias que interiorice su conocimiento.

En las Olimpiadas de Biología el utilizar diferentes estrategias metodológicas es importante porque con ello se logra una mejor calidad, eficiencia y eficacia en el proceso de enseñanza- aprendizaje. Específicamente, las estrategias metodológicas más utilizadas en este evento son; analizar, observar y comparar información, desarrollar problemas y ejecutar prácticas de laboratorio. Estas estrategias formarán parte de la retroalimentación de conocimientos que adquieren los estudiantes, para posteriormente ser evaluados como parte de su progreso académico.

\section{Papel de los docentes en las OLICOCIBI}

En las Olimpiadas de Biología, uno de sus objetivos es incorporar un cambio en la campo de las ciencias de la educación secundaria costarricense, donde los docentes involucrados en este proyecto presenten una adecuada metodología pedagógica, además involucren responsabilidad, innovación, trabajar conjuntamente para adquirir conocimientos mediante diferentes experiencias. Este enriquecimiento del profesorado puede ser usado luego como retroalimentación de conocimientos a los estudiantes que se interesan en participar en estas competencias.

Según el Colegio Hebreo Dr. Jaim Weitzman - CPEIP- (sin fecha), indica que para que una actividad sea esencialmente pedagógica, requiere que esta tenga: 
Sentido, esencia y conciencia de su propio rumbo y, por cierto, de su fin. Así, entonces, todas las actividades, la solución de problemas, la realización de proyectos, la exploración del entorno o la investigación de hechos nuevos configurarán un aprendizaje significativo y rico, plasmado de posibilidades valorizables. Tal aprendizaje estará fundamentado en la experiencia de los educandos, en situaciones vividas realmente, en conductas éticas no "enseñadas" sino fraguadas en su propia existencia.

Se trata de que el docente que participe en este tipo de competencias responda a un perfil, donde su función no sólo se base en la trasmisión de conocimientos, sino también debe orientar a los estudiantes para que aprendan a aprender. Para ello, es vital que a los profesores se les dé una formación continua, donde se les esté brindando capacitaciones, y así ser estimulados permanentemente, para facilitar el desarrollo de diferentes métodos o técnicas de enseñanza. En ese mismo hilo temático, poder tener la capacidad de trabajar en grupo, ser entusiastas, investigativos, reflexivos, participativos, poseer una excelente comunicación, motivación, con actitud positiva y sobre todo responsables en sus compromisos pedagógicos.

De acuerdo con las categorías que asigna el MEP a los educadores, el grado profesional MT5 corresponde a licenciatura o maestría de educación superior, es por ello que si el docente del área de las ciencias biológicas cuenta con este perfil, da mayor respaldo educativo. Según Pereira (2011), concuerda con lo expuesto anteriormente, en una encuesta realizada en el 2010 a 40 docentes participantes en las Olimpiadas, reveló que la mayoría cuenta con el grado profesional MT5, ver figura 2. 
Figura 2: Categorías profesionales de los y las docentes participantes en la fase final de la OLICOCIBI 2010

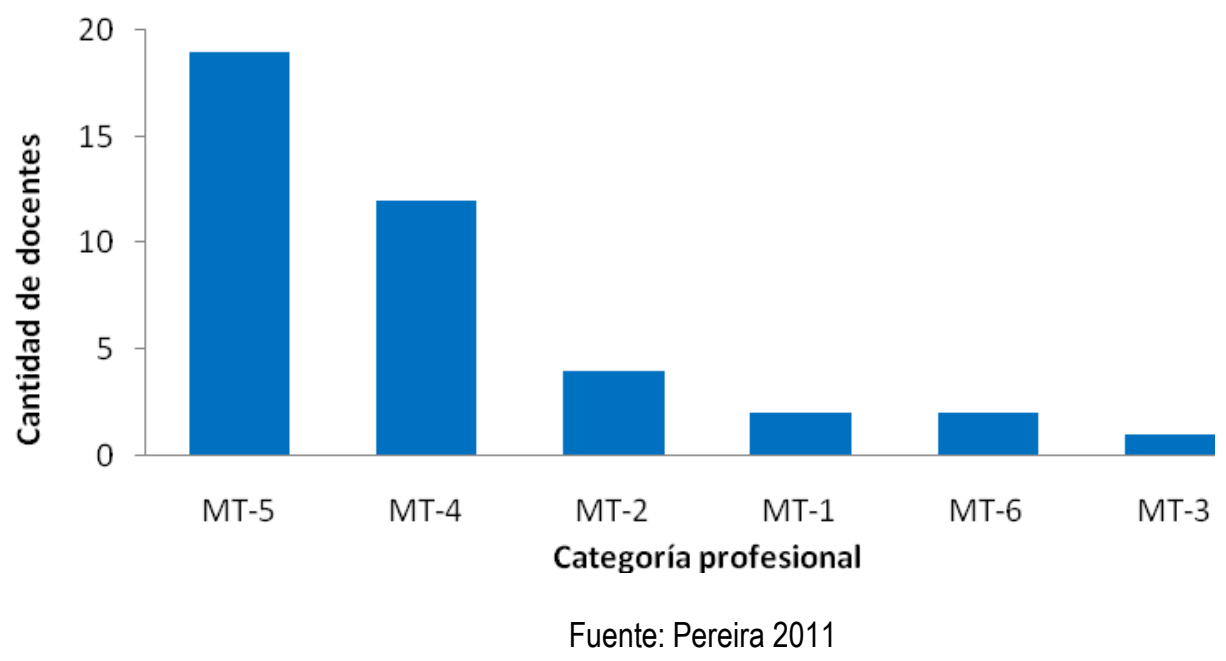

Las capacitaciones o talleres que brinda el comité organizador de las Olimpiadas de Biología a los docentes de secundaria tienen como objetivo promover diferentes métodos pedagógicos, así como refrescar y ampliar conocimientos en los temas que ellos deben desarrollar en los colegios. También, dar a conocer temas de interés actual en el área de las ciencias biológicas, al estar la ciencia vinculada con la tecnología es factible encontrar muchos cambios a corto plazo. De ahí que las ideas que adquieren los docentes mediante estas capacitaciones tienen el propósito de facilitar la trasmisión de conocimientos a los estudiantes para un mejor entendimiento del proceso de enseñanza en el área de la biología e incentivar a ellos en los avances científicos y tecnológicos.

Según Pereira (2011), a lo largo de los 5 años que tiene las Olimpiadas de Biología de estar realizándose, se han brindado una serie de talleres o capacitaciones a los docentes, algunos ejemplos de estas son:

- Taller Alfabetizando Científicamente a través de las OLICOCIBI.

- Taller de Actualización en Botánica General. 
- Taller de Actualización en el Mundo Vegetal.

- Taller de Biología Celular y Aplicaciones Biotecnológicas.

- Taller de Enseñanza Aprendizaje de la Biología.

- Taller de Estado de la Educación Biológica en Costa Rica.

- Taller de Importancia de la Biología: Ciencia, Tecnología y Sociedad.

- Taller de Técnicas Novedosas en Biología Molecular.

- Taller Evolución de Ecosistemas Marino-Costeros.

- Taller Actualización en Ecología.

Al ser los docentes uno de los principales inspiradores para motivar a los estudiantes, esto implica que ellos estén realizando diferentes estrategias metodológicas para ayudar a los estudiantes a asimilar su proceso de enseñanza y aprendizaje. Por lo que se resalta lo citado por Barzal y Ortíz (2006), donde señalan que:

Es necesario la participación de profesores que tengan buenas ideas como una acción renovadora orientada hacia la mejora educativa, y esto únicamente se llevará a cabo con la activa participación de docentes, que sean capaces de comprender y que deseen conducir cambios reales en las aulas e instituciones. 


\section{Conclusiones}

Las Olimpiadas de Biología se presentan como un evento que pretende ser renovador, continúo y lograr creatividad en los procesos académicos.

$>$ Los altos niveles de interés y participación de la comunidad educativa secundaria costarricense en estas competencias han provocado un indiscutible impacto social, proyectándose como una actividad de gran alcance y significado para el desarrollo de las Ciencias Biológicas en la educación.

> Realizar anualmente cursos de capacitación en las diferentes regionales del MEP a los docentes del área de las Ciencias fortalece sus estrategias metodológicas a la hora de llevar a cabo el proceso de enseñanza y aprendizaje.

$>$ Parte de las estrategias a utilizar con los estudiantes y profesores que participan en estas competencias es lograr motivar, tener buena comunicación, una actitud positiva, investigativa y participativa en el desarrollo de la actividad.

> La OLICOCIBI a través de pruebas teóricas como prácticas que imparte a los estudiantes ganadores de las olimpiadas nacionales busca solventar vacíos conceptuales que arrastran los estudiantes, y así, se geste la integración de contenidos conceptuales, procedimentales y actitudinales en el campo de la biología para lograr llevar representantes de alto nivel a las Olimpiadas Iberoamericanas.

$>$ El concentrar grupos tanto de estudiantes como profesores que comparten a nivel cognitivo, estrechen lazos de amistad, responsabilidad, trabajo en equipo, solidaridad, respeto y estímulo del pensamiento crítico científico permite de una manera efectiva ampliar los conocimientos tanto teóricos como prácticos de la biología.

$>$ Por medio de la divulgación y con la ayuda de todas las instituciones que participan en este proyecto, se pretende ampliar cada año más la 
participación de centros educativos públicos y privados de todo el país en las Olimpiadas Costarricenses de Ciencias Biológicas.

Las capacitaciones que se le brinda a los profesores como a los estudiantes fortalecen la incorporación de nuevas estrategias metodológicas y la adquisición de material didáctico actualizado.

> La OLICOCIBI establece puentes de comunicación entre el sistema de educación secundaria con el sistema de estudios superiores para fortalecer la enseñanza de las ciencias biológicas.

$>$ Este tipo de proyecto incentiva el intercambio cultural, social y cognitivo, entre estudiantes y profesores de diversos centros educativos de todo el país.

\section{Bibliografía}

Berzal, M y Ortiz, M. (2006). El papel de las reuniones científicas y educativas en la promoción de la investigación, la innovación y la formación del profesorado de biología. Interaccionar para cambiar y renovar: Revista de Educación en Biología. 9(1), pp. 6-13.

Camacho, S. (2011). Aportes del proyecto OLICOCIBI en la alfabetización científica de sus participantes en sus dimensiones conceptual, procedimental y actitudinal. Tesis de licenciatura. Universidad Nacional. Heredia, Costa Rica.

Colegio Hebreo Dr. Jaim Weitzman (CPEIP). (Sin fecha). Estrategias metodológicas. Educrea, centro de documentación. Recuperado de http://www.educrea.cl/documentacion/articulos/didactica/19_estrategias_me todologicas.html

Escuela de Ciencias Biológicas. (2007). Proyecto Olimpiada Costarricense de Biología.Universidad Nacional, Heredia, Costa Rica.

Escuela de Ciencias Biológicas. (2008). Proyecto Fortalecimiento y Consolidación de los Programas de Olimpiadas Costarricenses de Ciencias Biológicas e Internacionales de Biología, para mejorar la formación de los y las 
estudiantes preuniversitarias y los y las estudiantes universitarias con interés y afinidades hacia las Ciencias Biológicas.Universidad NacionalHeredia, Costa Rica.

Escuela de Ciencias Biológicas. (2009). Proyecto Fortalecimiento y Consolidación de los Programas de Olimpiadas Costarricenses de Ciencias Biológicas e Internacionales de Biología, para mejorar la formación de los y las estudiantes preuniversitarias y los y las estudiantes universitarias con interés y afinidades hacia las Ciencias Biológicas.Universidad NacionalHeredia, Costa Rica.

Digión, L; Sosa, M y Velásquez, I. (2006). Estrategias para la mediación pedagógica en ambientes de educación a distancia. Universidad Nacional de Santiago del Este. Argentina: Departamento de informática, Facultad de Ciencias Exactas y Tecnología.

Olimpiadas Costarricenses de Ciencias Biológicas. (Sin fecha).Propósitos. Universidad Nacional. Recuperado dehttp://www.una.ac.cr/olicocibi/index.php?option=com_content\&task=view \&id $=14 \&$ Itemid $=32$

Olimpiadas Costarricenses de Ciencias Biológicas. (Sin fecha).Antecedentes. Universidad Nacional. Recuperado de

http://www.una.ac.cr/olicocibi/index.php?option=com_content\&task=view\&id=13\&lt emid $=33$

Perdomo, M. (2008). Elrol y el perfil del docente en la educación a distancia. Docente descrita a la Universidad Centro-occidental "Lisandro Alvarado". Barquisimeto, Venezuela. Recuperado de http://www.salvador.edu.ar/vrid/publicaciones/PErdomo.doc

Pereira, J. (Diciembre, 2011). Presentación del informe final a la comisión organizadora de las Olimpiadas Costarricenses de Ciencias Biológicas. [Power Point]Heredia, Costa Rica: Universidad Nacional, Universidad Estatal a Distancia y Universidad de Costa Rica.

Soler, L; Fleota. A; Carenton, J; Cuello, P; Pérez, P y Palacios, M. (2006). El uso de una especie en extinción como herramienta en Educación Ambiental: experiencias pedagógicas en la Provincia de Corrientes, Argentina. Revista de Educación Biología 9(2), pp. 29-35. 